From the Fox Chase Cancer Center: Thomas Jefferson University, Philadelphia; Medical Oncology Associates, Kingston; Immunicon Corp, Huntingdon Valley, PA; the Dutch Colorectal Cance Group; Radboud University Nijmegen Medical Center, Nijmegen, the Netherlands; Hematology Oncology Associates, Port Saint Lucie, FL; Medical Oncology and Hematology PC, New Haven, CT; Union Hospital, Canton, $\mathrm{OH}$; Washington University, St Louis, MO; and Duke University Medical Center, Durham, NC.

Submitted December 26, 2007: accepted March 19, 2008.

Supported by Immunicon Corporation.

Presented in part at the 43rd Annual Meeting of the American Society of Clinical Oncology, June 1-5, 2007

Chicago, IL.

Authors' disclosures of potential conflicts of interest and author contributions are found at the end of this article.

Clinical Trials repository link available on JCO.org

Corresponding author: Steven J. Cohen, MD, Department of Medical Oncology, Fox Chase Cancer Center 333 Cottman Ave, Philadelphia, PA 19111; e-mail: S_Cohen@fccc.edu.

(C) 2008 by American Society of Clinical Oncology

0732-183X/08/2619-3213/\$20.00 DOI: $10.1200 / J C O .2007 .15 .8923$

\title{
Relationship of Circulating Tumor Cells to Tumor Response, Progression-Free Survival, and Overall Survival in Patients With Metastatic Colorectal Cancer
}

Steven J. Cohen, Cornelis J.A. Punt, Nicholas Iannotti, Bruce H. Saidman, Kert D. Sabbath, Nashat Y. Gabrail, Joel Picus, Michael Morse, Edith Mitchell, M. Craig Miller, Gerald V. Doyle, Henk Tissing, Leon W.M.M. Terstappen, and Neal J. Meropol

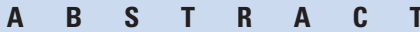

\section{Purpose}

As treatment options expand for metastatic colorectal cancer (mCRC), a blood marker with a prognostic and predictive role could guide treatment. We tested the hypothesis that circulating tumor cells (CTCs) could predict clinical outcome in patients with mCRC.

\section{Patients and Methods}

In a prospective multicenter study, CTCs were enumerated in the peripheral blood of 430 patients with $\mathrm{mCRC}$ at baseline and after starting first-, second-, or third-line therapy. CTCs were measured using an immunomagnetic separation technique.

\section{Results}

Patients were stratified into unfavorable and favorable prognostic groups based on CTC levels of three or more or less than three CTCs/7.5 mL, respectively. Patients with unfavorable compared with favorable baseline CTCs had shorter median progression-free survival (PFS; $4.5 \vee 7.9$ months; $P=.0002$ ) and overall survival (OS; $9.4 \vee 18.5$ months; $P<.0001$ ). Differences persisted at 1 to 2,3 to 5,6 to 12, and 13 to 20 weeks after therapy. Conversion of baseline unfavorable CTCs to favorable at 3 to 5 weeks was associated with significantly longer PFS and OS compared with patients with unfavorable CTCs at both time points (PFS, $6.2 \vee 1.6$ months; $P=.02$; OS, $11.0 \vee 3.7$ months; $P=.0002$ ). Among nonprogressing patients, favorable compared with unfavorable CTCs within 1 month of imaging was associated with longer survival (18.8 v 7.1 months; $P<.0001$ ). Baseline and follow-up CTC levels remained strong predictors of PFS and OS after adjustment for clinically significant factors.

\section{Conclusion}

The number of CTCs before and during treatment is an independent predictor of PFS and OS in patients with metastatic colorectal cancer. CTCs provide prognostic information in addition to that of imaging studies.

\section{J Clin Oncol 26:3213-3221. (c) 2008 by American Society of Clinical Oncology}

\section{INTRODUCTION}

Colorectal cancer is the second leading cause of cancer death in the United States, with approximately 154,000 new cases and 52,000 deaths expected in 2007. ${ }^{1}$ The number of therapeutic agents for metastatic colorectal cancer (mCRC) has increased during the last several years, with concomitant improvement in outcome. ${ }^{2-4}$ With three classes of cytotoxic agents and two classes of therapeutic antibodies, treatment decision making is more complicated. Treatment often includes aggressive therapy as well as treatment holidays. The ability to identify patients with worse prognosis or those destined to progress quickly could have broad clinical application.
The presence of circulating tumor cells (CTCs) was speculated since Recamier coined the term "metastasis" in 1829, ${ }^{5}$ and confirmed with Engell's documentation of cancer cells in the circulation in $1955 .{ }^{6}$ Recent refinement of an immunomagnetic separation technology to reliably and reproducibly isolate, enumerate, and characterize CTCs in epithelial malignancies ${ }^{7}$ has enabled further study of the CTC as a prognostic and predictive marker. We conducted a pilot study demonstrating that CTCs can be isolated and enumerated in patients with mCRC. ${ }^{8}$ We also noted that patients with disease progression had greater serial increases in CTC number than did nonprogressors. CTCs are present in the blood of patients with many cancers, but are extremely rare in healthy people. ${ }^{7}$ In patients with 
breast cancer, CTC number is an independent predictor of progression-free survival (PFS) and overall survival (OS). ${ }^{9}$ On the basis of this observation and our pilot study findings, ${ }^{8}$ we initiated this multicenter study to evaluate whether CTCs could serve as a prognostic and/or predictive marker in patients with mCRC.

\section{PATIENTS AND METHODS}

\section{Study Design}

This prospective trial was conducted at 55 clinical centers throughout the US, the Netherlands, and the United Kingdom to evaluate the agreement of CTC number with response by imaging and the ability of CTC number to predict PFS and OS in patients with $\mathrm{mCRC}$. Principal inclusion criteria were measurable mCRC initiating any first- or second-line systemic therapy or third-line therapy with an epidermal growth factor receptor inhibitor. All patients had an Eastern Cooperative Oncology Group (ECOG) performance status score of 0 to 2 and hemoglobin of at least $8 \mathrm{~g} / \mathrm{dL}$. The institutional review boards at each center approved the study protocol, and all patients provided written informed consent.

\section{Imaging}

Computed tomography or magnetic resonance imaging scans of the chest, abdomen and pelvis were to be performed at baseline and every 6 to 12 weeks after initiating treatment. Image interpretation was performed by a certified radiologist at each participating site using Response Evaluation Criteria in Solid Tumors (RECIST $)^{10}$ to classify each disease assessment as complete response (CR), partial response (PR), stable disease (SD), or progressive disease (PD). Patients who died before a follow-up imaging study were considered to have PD. For response to therapy at the first follow-up disease evaluation, the favorable group was defined as those with nonprogressive disease (NPD; including SD/PR/CR categories) and the unfavorable group as those with PD or death.

\section{Isolation and Enumeration of CTCs}

Peripheral blood was collected for CTC evaluation before the initiation of therapy (baseline) and subsequently at 1 to 2,3 to 5,6 to 12 , and 13 to 20 weeks after initiating treatment. Blood samples were drawn into $10-\mathrm{mL}$ evacuated tubes (CellSave, Immunicon, Huntingdon Valley, PA). Samples were maintained at room temperature, mailed overnight, and processed within 96 hours of collection. All CTC evaluations were performed without knowledge of patient clinical status in one of four central laboratories. The CellSearch System (Veridex LLC, Raritan, NJ) was used for CTC enumeration, the technical details of which, including accuracy, precision, linearity, and reproducibility have been previously described. ${ }^{7}$ CTCs were defined as EpCAM isolated intact cells staining positive for cytokeratin and negative for CD45. At each time point, the favorable and unfavorable groups were defined as those having CTC levels less than the selected threshold or greater than or equal to the selected threshold, respectively. Two tubes of blood for CTCs were drawn at each time point to assess intrapatient reproducibility and confirmed a strong correlation between the two tubes $\left(\mathrm{R}^{2}=0.96\right.$; Appendix Figs A1A and A1B, online only).

\section{Statistical Analysis}

The primary objective was assessment of agreement of CTCs after the initiation of therapy with response to therapy. We predicted that approximately $20 \%$ of patients would have unfavorable CTC levels after initiation of therapy. The sample size was calculated to provide adequate power for evaluation of the primary and secondary (association of CTCs with PFS and OS) objectives. For the primary objective, agreement was defined as favorable CTC corresponding with NPD or unfavorable CTC corresponding with PD. A one-group $\chi^{2}$ test with a one-sided $P$ value of .025 would have $80 \%$ power to reject a null hypothesis of less than $60 \%$ overall agreement between CTC and the response to therapy as determined by imaging with a sample size of 78 assessable patients and an alternative hypothesis of at least $75 \%$ agreement. A significance level of .025 was used because an interim analysis was planned for selection of the optimal blood draw time point and CTC threshold. For the secondary objective, it was assumed that patients with favorable CTCs would have a median PFS and OS twice that of patients with unfavorable CTCs, equating to a hazard ratio of 2.0. A log-rank test for equality of survival curves with a one-sided $P$ value of .05 would have $80 \%$ power to detect a difference between the PFS and OS curves of patients with unfavorable and favorable CTCs, with a minimum of 37 patients in each group. Therefore, we initially planned enrollment of 200 patients, with an interim review planned after enrollment of 100 patients.

Based on a predetermined protocol interim analysis, the first 109 patients enrolled (training set) were used to select the optimal blood draw time point and a CTC cutoff for the stratification of patients into favorable and unfavorable prognostic groups based on the CTC counts after initiation of therapy. To select the optimal blood draw time point after the initiation of therapy, receiver operating characteristic curve analysis was used, the results of which led to selection of the 3- to 5-week blood draw time point because it provided the largest area under the receiver operating characteristic curve (75\%). Thresholds of 1 to 10 for the 3- to 5-week CTC levels were systematically correlated with imaging, and the percentage CTC positive, sensitivity, specificity, and overall agreement for comparison of CTC and response at the first follow-up imaging study were evaluated. A threshold of at least three CTCs was chosen based on these results (data not shown). Using this threshold, approximately $10 \%$ of patients had unfavorable CTC counts at 3 to 5 weeks, requiring an increased enrollment target of 400 patients.

The selected threshold was then validated using the last 321 patients enrolled (validation set). Distribution of patients above and below the threshold level in the training and validation sets was compared using Fisher's exact test. The median patient ages and years to metastasis were compared using the nonparametric $k$-sample $\chi^{2}$ test for equality of the medians.

Patients were followed for progression by imaging every 6 to 12 weeks and after progression for overall survival every 6 months for up to 2 years. The study was monitored by an independent clinical research organization. PFS was defined as the elapsed time from blood collection to progression or death. OS was defined as the elapsed time from blood collection to death. Patients were censored at last follow-up if PD or death had not occurred. Separate Kaplan-Meier survival plots were generated on the basis of CTC levels at baseline and follow-up blood collections. Survival curves were compared using log-rank testing. Cox proportional hazards regression was used to determine univariate and multivariate hazards ratios for PFS and OS.

\section{RESULTS}

\section{Patient Characteristics}

Between February 2004 and November 2006 a total of 481 patients were enrolled, 430 of whom met inclusion and exclusion criteria and were assessable for the primary and/or secondary objectives. At the time of these analyses, death had occurred in 202 (47\%) of the 430 patients, with a mean follow-up time for the 228 (53\%) patients still alive of $12.6 \pm 6.5$ months (median, 11.0 months; range, 0.8 to 30.0 months). Appendix Figure A2 (online only) summarizes the number of patients assessable for evaluation of the primary and secondary objectives and reasons for exclusion. Patient characteristics are listed in Table 1.

\section{CTC at Baseline}

At baseline, $26 \%$ of patients had unfavorable CTCs ( $\geq$ three CTCs/7.5 mL of blood). Patients with liver metastases and poorer performance status had higher baseline CTC levels (Table 2).

\section{Comparison of Training and Validation Sets}

Comparison of the results from training and validation sets demonstrated no significant differences in the percentage of patients with unfavorable CTCs at 3 to 5 weeks $(16 \% v 10 \%$, respectively; $P=.232)$ 


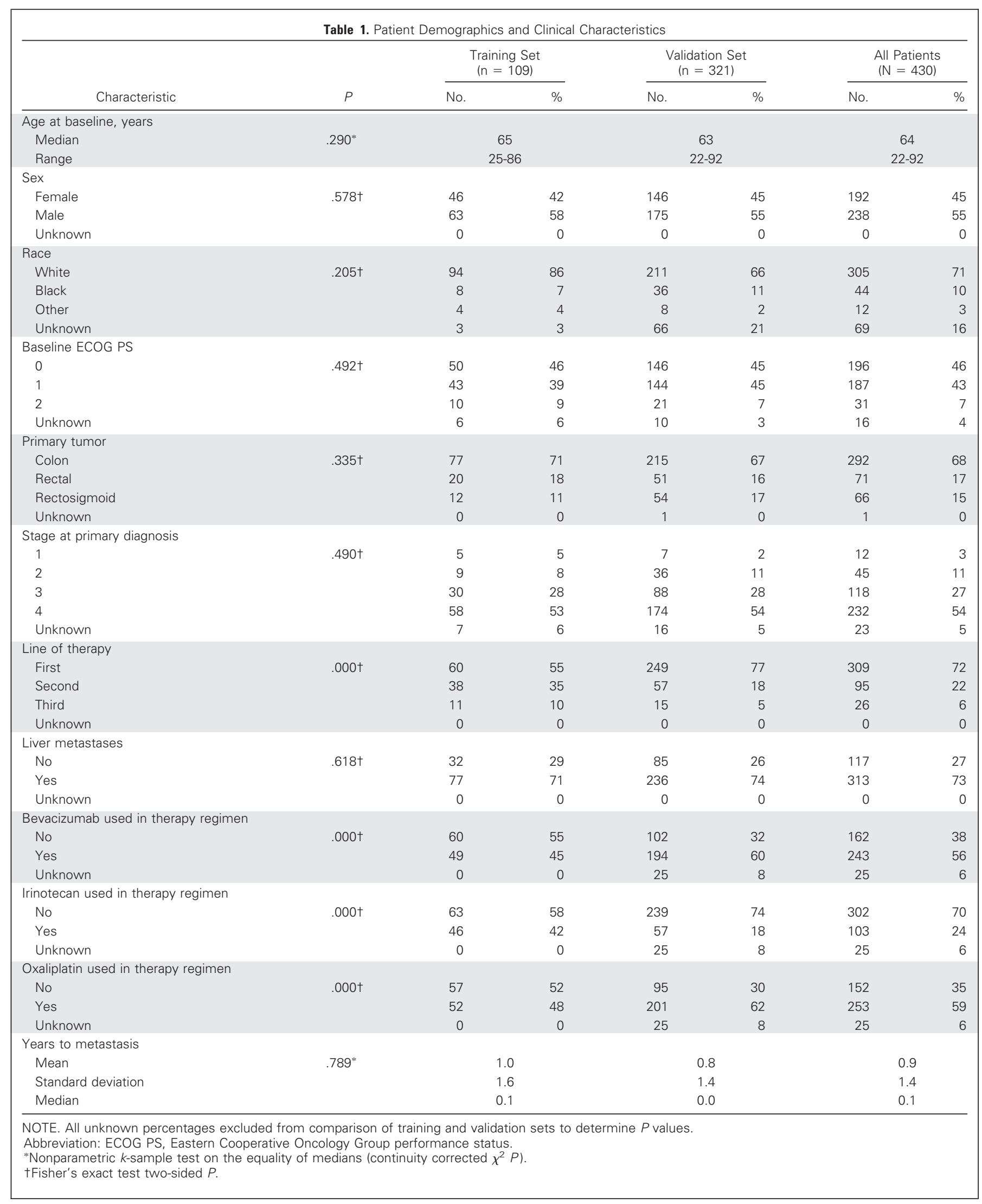




\begin{tabular}{|c|c|c|c|c|c|c|c|c|}
\hline Patient Subset & $\geq 1$ & $\geq 2$ & $\geq 3$ & $\geq 4$ & $\geq 5$ & $\geq 10$ & $\geq 100$ & $\geq 250$ \\
\hline \multicolumn{9}{|l|}{ Metastatic colorectal cancer $(n=430)$} \\
\hline All with baseline draw $(n=413)$ & 48 & 33 & 26 & 22 & 18 & 12 & 1 & 0 \\
\hline \multicolumn{9}{|l|}{ Line of therapy ( $n=413$ ) } \\
\hline First $(n=296)$ & 44 & 31 & 24 & 20 & 17 & 11 & 1 & 0 \\
\hline Fisher's exact $P$ & .093 & .203 & .334 & .275 & .497 & .194 & .026 & .063 \\
\hline \multicolumn{9}{|l|}{ Primary tumor type $(n=413)$} \\
\hline Colon $(n=278)$ & 50 & 35 & 28 & 23 & 19 & 12 & 1 & 0 \\
\hline Rectal $(n=70)$ & 41 & 31 & 27 & 24 & 20 & 14 & 3 & 0 \\
\hline Rectosigmoid $(n=65)$ & 43 & 29 & 17 & 14 & 12 & 9 & 0 & 0 \\
\hline Fisher's exact $P$ & .329 & .672 & .181 & .228 & .413 & .660 & .249 & $>.99$ \\
\hline Fisher's exact $P$ & .227 & .014 & .026 & .010 & .019 & .067 & .264 & .073 \\
\hline \multicolumn{9}{|l|}{ Site of metastases ( $n=413$ ) } \\
\hline Liver involvement $(n=302)$ & 54 & 39 & 30 & 25 & 21 & 13 & 1 & 0 \\
\hline No liver involvement $(n=111)$ & 30 & 18 & 14 & 14 & 10 & 8 & 1 & 0 \\
\hline Fisher's exact $P$ & .000 & .000 & .001 & .015 & .009 & .225 & $>.99$ & $>.99$ \\
\hline
\end{tabular}

or in the percentage agreement with imaging (77\% $v 78 \%$, respectively; $P=.878$ ). To further evaluate combining training and validation sets, sites within the same geographic regions with fewer than 10 assessable patients enrolled were grouped and analyzed as one site. No significant differences were noted between training and validation sets with either the percentage of unfavorable CTC samples at 3 to 5 weeks $(P=.633)$ or in the percentage agreement with imaging $(P=.728)$. Therefore, for evaluation of primary and secondary outcomes, data from the training and validation sets were pooled and a CTC threshold of at least three CTCs/7.5 mL was utilized.

\section{Relationship of CTC to Radiographic Imaging}

Of 430 assessable patients, 384 (89\%) had a follow-up imaging study performed and assessed using RECIST (time to first imaging study: mean, $9.2 \pm 2.8$ weeks; median, 8.7 weeks). Twenty-eight (7\%) did not have a follow-up imaging study performed, and $18(4 \%)$ died before a follow-up imaging study. A total of 334 patients (78\%) had a
CTC assessment 3 to 5 weeks after starting therapy (mean, $3.8 \pm 0.7$ weeks; median, 4.0 weeks). Three hundred twenty patients were included in the analysis of the primary objective because they had a follow-up imaging study analyzed by RECIST, or they died before a follow-up imaging study and they had CTCs assessed 3 to 5 weeks after initiation of therapy. In this subset of 320 patients, three (1\%) had a CR, 87 (27\%) had a PR, 156 (49\%) had SD, and 68 (21\%) had PD at their first follow-up imaging study. Six (2\%) died before a follow-up imaging study.

In 74 patients (23\%) with PD or death, $20(27 \%)$ had unfavorable CTCs at 3 to 5 weeks compared with 18 (7\%) with NPD. Overall, CTC had a sensitivity of $27 \%$ ( $95 \%$ CI, $17 \%$ to $39 \%$ ), specificity of $93 \%$ (95\% CI, $89 \%$ to $96 \%$ ), a positive predictive value of 53\% (95\% CI, $36 \%$ to $69 \%$ ), a negative predictive value of $81 \%$ (95\% CI, $76 \%$ to $85 \%)$, and overall agreement or accuracy of $78 \%$ (95\% CI, $73 \%$ to $82 \%)$. Table 3 summarizes the comparison of imaging response and CTC at 3 to 5 weeks.

Table 3. Response to Imaging $v$ CTC Category at 3-5 Weeks

\begin{tabular}{|c|c|c|c|c|c|c|}
\hline \multirow[b]{3}{*}{ Response to Therapy by Imaging (RECIST criteria) } & \multicolumn{4}{|c|}{$\begin{array}{c}\text { CTCs 3-5 Weeks After the Initiation of } \\
\text { Therapy }\end{array}$} & \multirow[b]{3}{*}{ Total } & \multirow[b]{3}{*}{$\%$ of Total Set } \\
\hline & \multicolumn{2}{|c|}{$<3$ CTCs } & \multicolumn{2}{|c|}{$\geq 3 \mathrm{CTCs}$} & & \\
\hline & No. & $\%$ & No. & $\%$ & & \\
\hline Nonprogressive disease (stable disease, partial or complete response) & 228 & 93 & 18 & 7 & 246 & 77 \\
\hline Progressive disease (or death) & 54 & 73 & 20 & 27 & 74 & 23 \\
\hline Total & 282 & 88 & 38 & 12 & 320 & 100 \\
\hline
\end{tabular}

Abbreviations: CTC, circulating tumor cell; RECIST, Response Evaluation Criteria in Solid Tumors. 


\section{CTC As a Prognostic Marker}

The median PFS for the 430 assessable patients was 7.2 months (95\% CI, 6.7 to 7.9 months) and median OS was 15.5 months (95\% CI, 14.0 to 18.4 months). Patients with unfavorable CTCs at baseline had a significantly shorter median PFS (4.5 months; 95\% CI, 3.7 to 6.3 months) and median OS (9.4 months; $95 \%$ CI, 7.5 to 11.6 months) compared with patients with less than three CTCs/7.5 mL (median PFS, 7.9 months; 95\% CI, 7.0 to 8.6 months; median OS, 18.5 months; 95\% CI, 15.5 to 21.2 months; Fig $1 \mathrm{~A}$ and $1 \mathrm{~B})$.

\section{CTC As a Predictive Marker}

The predictive value of CTC after 1 to 2, 3 to 5, 6 to 12 , and 13 to 20 weeks of treatment is shown in Figure 1C and 1D. PFS and OS were significantly shorter at all time points for patients with at least three CTCs during therapy compared with those with fewer than three CTCs. Patients with PD before the time of the blood draw evaluation were excluded from the PFS analysis at that time point. Figure A3A and $\mathrm{A} 3 \mathrm{~B}$ (online only) show the median OS for increasing CTC thresholds at baseline and 3 to 5 weeks, demonstrating a plateau in OS at the three-CTC threshold.

Figure 1E and $1 \mathrm{~F}$ shows the relationship of CTC change from baseline to 3 to 5 weeks and clinical outcome in 319 patients. KaplanMeier plots are generated for those patients with CTCs who remained favorable (group 1), CTCs that remained unfavorable (group 4), or those who converted to the unfavorable (group 3) or favorable groups (group 2). Four (1\%) of these patients showed evidence of PD before the date of the follow-up CTC evaluation and were excluded from the PFS analysis. Median PFS for 226 patients (72\%) with favorable CTC at both time points (group 1) was not significantly different from that of the 52 patients (16\%) who converted from the unfavorable to the favorable CTC group (group 2). However, the median PFS of these 52 patients (group 2) was significantly longer compared with that of the 28 patients who had unfavorable CTCs at both time points (group 4, Fig 1E). The median OS of 227 patients with favorable CTCs at both time points (group 1) was significantly longer compared with that of the 53 patients who began with unfavorable CTCs but converted to favorable CTCs at 3 to 5 weeks (group 2; Fig 1F). The median OS for the 53 patients $(17 \%)$ that began in the unfavorable group but converted to the favorable group (group 2) was significantly longer compared with that of patients who remained with unfavorable CTCs at both time points (group 4, Fig 1F).

\section{Predictors of PFS and OS}

In univariate Cox regression analyses, age, line of therapy, type of therapy, ECOG performance status, and CTC levels (at baseline and all follow-ups) were significantly associated with both PFS and OS. For multivariate Cox regression analyses, only the univariately significant clinical factors for the time point being evaluated were included in the multivariate model for that particular time point. After adjusting for these clinically significant factors, CTCs at baseline and all follow-up time points remained strong predictors of PFS and OS (Table 4).

\section{Relationship of CTCs and Imaging to OS}

Figure 2A shows that the OS for 121 patients (30\%) with CR or $\mathrm{PR}$ at first imaging was significantly longer compared with 186 patients $(46 \%)$ with stable disease and 95 patients (20\%) with PD or death. A total of 364 patients had a CTC level determined within 1 month of the follow-up imaging study or death. Figure 2B demon- strates that the OS of 335 patients (92\%) with favorable CTCs at the first follow-up imaging study was significantly longer compared with the OS of 29 patients ( $8 \%$ ) with unfavorable CTCs. Figure 2C shows that the OS of 271 patients $(74 \%)$ with NPD and favorable CTCs at the first follow-up imaging study (group 1) was significantly longer than that of 64 patients (18\%) with PD and favorable CTCs (group 2), 13 patients (4\%) with unfavorable CTCs and NPD (group 3), and 16 patients (4\%) with PD and unfavorable CTCs (group 4). The OS of patients with PD and favorable CTCs (group 2) was significantly longer compared with patients with PD and unfavorable CTCs (group 4).

\section{DISCUSSION}

Building on our pilot study, this current multicenter study demonstrates that CTCs can serve as both a prognostic and predictive factor for patients with mCRC. The presence of at least three CTCs at baseline and follow-up is a strong independent prognostic factor for inferior PFS and OS. When utilized in conjunction with imaging studies, CTCs provide additional prognostic information.

There are several scenarios for which CTCs could have utility in colorectal cancer. The data presented suggest that CTCs may be used as a stratification factor in future advanced disease treatment studies. The current list of validated prognostic factors is short, with only performance status being universally recognized. ${ }^{11,12}$ Further study should prospectively address whether modification of treatment based on unfavorable CTCs early in the course of treatment will result in improvement in PFS or OS. As treatment has become more effective for mCRC, decision making has become more complicated. Five classes of drugs are available for treatment, and traditional definitions of lines of therapy have blurred. ${ }^{13}$ The most common initial chemotherapy backbone is a fluoropyrimidine with oxaliplatin or irinotecan. CTC levels drawn at 3 to 5 weeks and 6 to 12 weeks, before typical imaging intervals, may have the potential to inform treatment choices and spare patients unnecessary toxicity by suggesting that an early change in therapy is warranted.

Patients with mCRC who respond to initial chemotherapy are often considered for reduction in treatment intensity ${ }^{14}$ or a treatment break. ${ }^{15}$ A potential disadvantage of this approach is rapid disease progression during treatment holidays. CTCs may have a role in identifying patients who could safely have prolonged treatment breaks versus those who need to resume therapy more quickly. It is also plausible that CTCs could be utilized to assist management of earlierstage colorectal cancer patients. Finally, CTC phenotyping could demonstrate characteristics to select patients for targeted therapies, a scenario that has begun to be formally tested in the clinic. ${ }^{16}$ The above scenarios will require prospective study to define the role for CTCs.

Limitations of this study must be considered. Patients undergoing various lines of therapy were included, which may influence the ability to generalize results to any one group. Patients also had flexibility regarding the exact dates of blood draws and computed tomography scans. However, the timeframes were well defined, and this study design more accurately reflects everyday clinical practice. Finally, the percentage of patients with unfavorable CTCs at baseline (26\%) and overall CTC yield is less than in other epithelial malignancies such as breast cancer. 


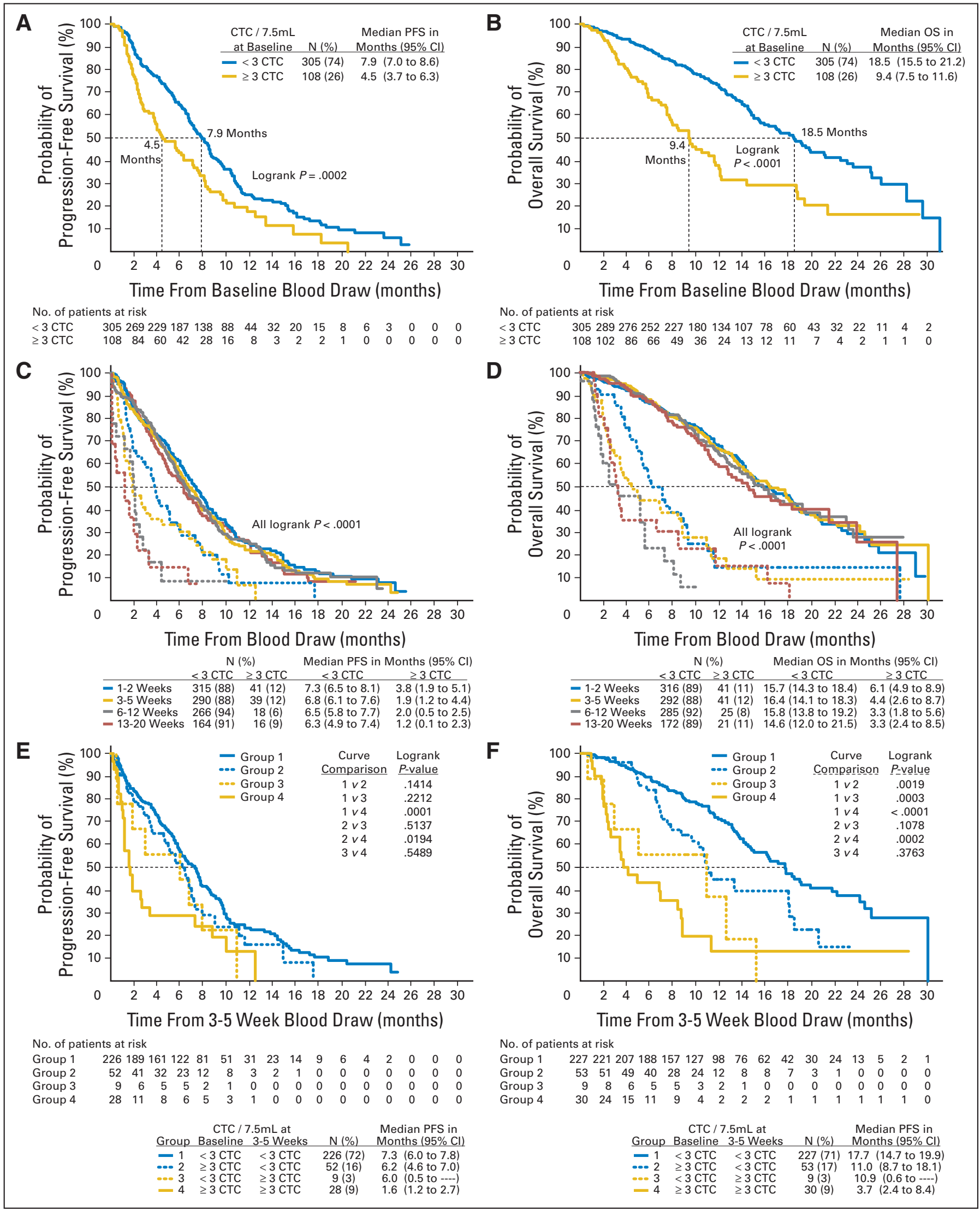

Fig 1. Progression-free survival (PFS) and overall survival (OS) of metastatic colorectal cancer patients with $<$ three and $\geq$ three circulating tumor cells (CTCs) in $7.5 \mathrm{~mL}$ of blood (A, B) before therapy, (C, D) 1 to 2, 3 to 5, 6 to 12, and 13 to 20 weeks after initiation of therapy, and $(E, F)$ by circulating tumor cell status at baseline and 3 to 5 weeks. 


\begin{tabular}{|c|c|c|c|c|c|c|c|c|c|c|}
\hline \multirow[b]{2}{*}{ Parameter } & \multicolumn{2}{|c|}{ Categories } & \multicolumn{4}{|c|}{ PFS Risk From Blood Draw } & \multicolumn{4}{|c|}{ OS Risk From Blood Draw } \\
\hline & Positive & Negative & HR & $95 \% \mathrm{Cl}$ & $P^{*}$ & $\begin{array}{l}\text { No. of } \\
\text { Patients }\end{array}$ & $\mathrm{HR}$ & $95 \% \mathrm{Cl}$ & $P^{*}$ & $\begin{array}{l}\text { No. of } \\
\text { Patients }\end{array}$ \\
\hline Analysis using baseline CTC count & & & & & & 373 & & & & 373 \\
\hline Baseline CTC No. & $>3$ & $<3$ & 1.74 & 1.33 to 2.26 & .000 & & 2.45 & 1.77 to 3.39 & .000 & \\
\hline Line of therapy & $2 n d$ or $3 r d$ & $1 \mathrm{st}$ & 1.73 & 1.32 to 2.28 & .000 & & 1.55 & 1.10 to 2.18 & .012 & \\
\hline Age at baseline blood draw, years & $\geq 65$ & $<65$ & 1.42 & 1.12 to 1.81 & .004 & & 1.78 & 1.30 to 2.44 & .000 & \\
\hline ECOG status at study entry & \multicolumn{2}{|c|}{$2 \vee 1 \vee 0$} & 1.16 & 0.96 to 1.38 & .117 & & 1.47 & 1.16 to 1.86 & .001 & \\
\hline Bevacizumab used in treatment regimen? & Yes & No & 0.62 & 0.49 to 0.80 & .000 & & 0.66 & 0.48 to 0.91 & .011 & \\
\hline Irinotecan used in treatment regimen? & Yes & No & 0.75 & 0.51 to 1.10 & .146 & & 1.23 & 0.76 to 1.98 & .400 & \\
\hline Oxaliplatin used in treatment regimen? & Yes & No & 0.54 & 0.38 to 0.77 & .001 & & 0.95 & 0.61 to 1.49 & .831 & \\
\hline Analysis using 1- to 2-week CTC count & & & & & & 320 & & & & 321 \\
\hline 1- to 2-week CTC No. & $>3$ & $<3$ & 1.85 & 1.28 to 2.68 & .001 & & 2.90 & 1.92 to 4.36 & .000 & \\
\hline Line of therapy & 2 nd or $3 r d$ & $1 \mathrm{st}$ & 1.99 & 1.46 to 2.71 & .000 & & 1.73 & 1.19 to 2.51 & .004 & \\
\hline Age at baseline blood draw, years & $\geq 65$ & $<65$ & 1.47 & 1.13 to 1.91 & .004 & & 1.77 & 1.26 to 2.49 & .001 & \\
\hline ECOG Status at study entry & \multicolumn{2}{|c|}{$2 \vee 1 \vee 0$} & 1.25 & 1.03 to 1.53 & .026 & & 1.53 & 1.19 to 1.97 & .001 & \\
\hline Bevacizumab used in treatment regimen? & Yes & No & 0.62 & 0.48 to 0.81 & .000 & & 0.74 & 0.53 to 1.04 & .085 & \\
\hline Irinotecan used in treatment regimen? & Yes & No & 0.67 & 0.44 to 1.03 & .067 & & 1.26 & 0.75 to 2.12 & .387 & \\
\hline Oxaliplatin used in treatment regimen? & Yes & No & 0.50 & 0.34 to 0.75 & .001 & & 0.93 & 0.57 to 1.51 & .758 & \\
\hline Analysis using 3- to 5-week CTC count & & & & & & 297 & & & & 301 \\
\hline 3- to 5-week CTC No. & $>3$ & $<3$ & 2.30 & 1.56 to 3.38 & .000 & & 4.78 & 3.11 to 7.34 & .000 & \\
\hline Line of therapy & $2 n d$ or $3 r d$ & $1 \mathrm{st}$ & 1.88 & 1.38 to 2.56 & .000 & & 2.04 & 1.39 to 2.98 & .000 & \\
\hline Age at baseline blood draw, years & $\geq 65$ & $<65$ & 1.53 & 1.16 to 2.00 & .002 & & 1.92 & 1.37 to 2.70 & .000 & \\
\hline ECOG status at study entry & \multicolumn{2}{|c|}{$2 \vee 1 \vee 0$} & 1.16 & 0.95 to 1.41 & .153 & & 1.33 & 1.03 to 1.71 & .030 & \\
\hline Bevacizumab used in treatment regimen? & Yes & No & 0.65 & 0.49 to 0.86 & .003 & & 0.84 & 0.59 to 1.21 & .354 & \\
\hline Irinotecan used in treatment regimen? & Yes & No & 0.60 & 0.39 to 0.92 & .018 & & - & - & & \\
\hline Oxaliplatin used in treatment regimen? & Yes & No & 0.46 & 0.31 to 0.64 & .000 & & 0.85 & 0.61 to 1.18 & .332 & \\
\hline Analysis using 6- to 12 -week CTC count & & & & & & 263 & & & & 279 \\
\hline 6- to 12-week CTC No. & $>3$ & $<3$ & 3.64 & 2.10 to 6.30 & .000 & & 9.35 & 5.28 to 16.54 & .000 & \\
\hline Line of therapy & 2 nd or $3 r d$ & $1 \mathrm{st}$ & 1.78 & 1.26 to 2.52 & .001 & & 1.46 & 0.95 to 2.24 & .082 & \\
\hline Age at baseline blood draw, years & $\geq 65$ & $<65$ & 1.45 & 1.09 to 1.94 & .012 & & 1.58 & 1.08 to 2.32 & .017 & \\
\hline ECOG status at study entry & \multicolumn{2}{|c|}{$2 \vee 1 \vee 0$} & - & & - & & 1.56 & 1.17 to 2.08 & .003 & \\
\hline Bevacizumab used in treatment regimen? & Yes & No & 0.64 & 0.47 to 0.86 & .003 & & 0.83 & 0.56 to 1.25 & .381 & \\
\hline Irinotecan used in treatment regimen? & Yes & No & 0.73 & 0.47 to 1.14 & .168 & & - & - & & \\
\hline Oxaliplatin used in treatment regimen? & Yes & No & 0.55 & 0.37 to 0.83 & .004 & & - & - & & \\
\hline Analysis using 13 - to 20 -week CTC count & & & & & & 170 & & & & 193 \\
\hline 13- to 20-week CTC No. & $>3$ & $<3$ & 4.18 & 2.17 to 8.03 & .000 & & 4.01 & 2.11 to 7.62 & .000 & \\
\hline Line of therapy & \multicolumn{2}{|c|}{$2 n d$ or $3 r d$} & 1.78 & 1.12 to 2.83 & .014 & & 1.30 & 0.76 to 2.21 & .332 & \\
\hline Age at baseline blood draw, years & $\geq 65$ & $<65$ & 1.33 & 0.92 to 1.93 & .132 & - & - & & & \\
\hline Bevacizumab used in treatment regimen? & Yes & No & 0.68 & 0.46 to 1.00 & .047 & - & - & & & \\
\hline Oxaliplatin used in treatment regimen? & Yes & No & 0.86 & 0.59 to 1.26 & .445 & - & - & & & \\
\hline
\end{tabular}

In conclusion, this study demonstrates the independent prognostic and predictive value of CTCs for patients initiating chemotherapy for mCRC. The data obtained in this clinical trial supported US Food and Drug Administration clearance of the CellSearch system for enumeration of CTCs in $\mathrm{mCRC}$, and this test is now commercially available. Our study was not designed to assess whether a change in therapy based on unfavorable CTCs is beneficial. However, clinical trials to explore this hypothesis are warranted.

\section{AUTHORS' DISCLOSURES OF POTENTIAL CONFLICTS OF INTEREST}

Although all authors completed the disclosure declaration, the following author(s) indicated a financial or other interest that is relevant to the subject matter under consideration in this article. Certain relationships marked with a " $U$ " are those for which no compensation was received; those relationships marked with a " $C$ " were compensated. For a detailed description of the disclosure categories, or for more information about ASCO's conflict of interest policy, please refer to the Author Disclosure Declaration and the Disclosures of Potential Conflicts of Interest section in Information for Contributors.

Employment or Leadership Position: M. Craig Miller, Immunicon Corporation (C); Gerald V. Doyle, Immunicon Corporation (C); Henk Tissing, Immunicon Corporation (C); Leon W.M.M. Terstappen, Immunicon Corporation (C) Consultant or Advisory Role: None Stock Ownership: M. Craig Miller, Immunicon Corporation; Gerald V. Doyle, Immunicon Corporation; Henk Tissing, Immunicon Corporation; Leon W.M.M. Terstappen, Immunicon Corporation Honoraria: None

Research Funding: Steven J. Cohen, Immunicon Corporation; Cornelis J.A. Punt, Immunicon Corporation; Nicholas Iannotti, Immunicon 


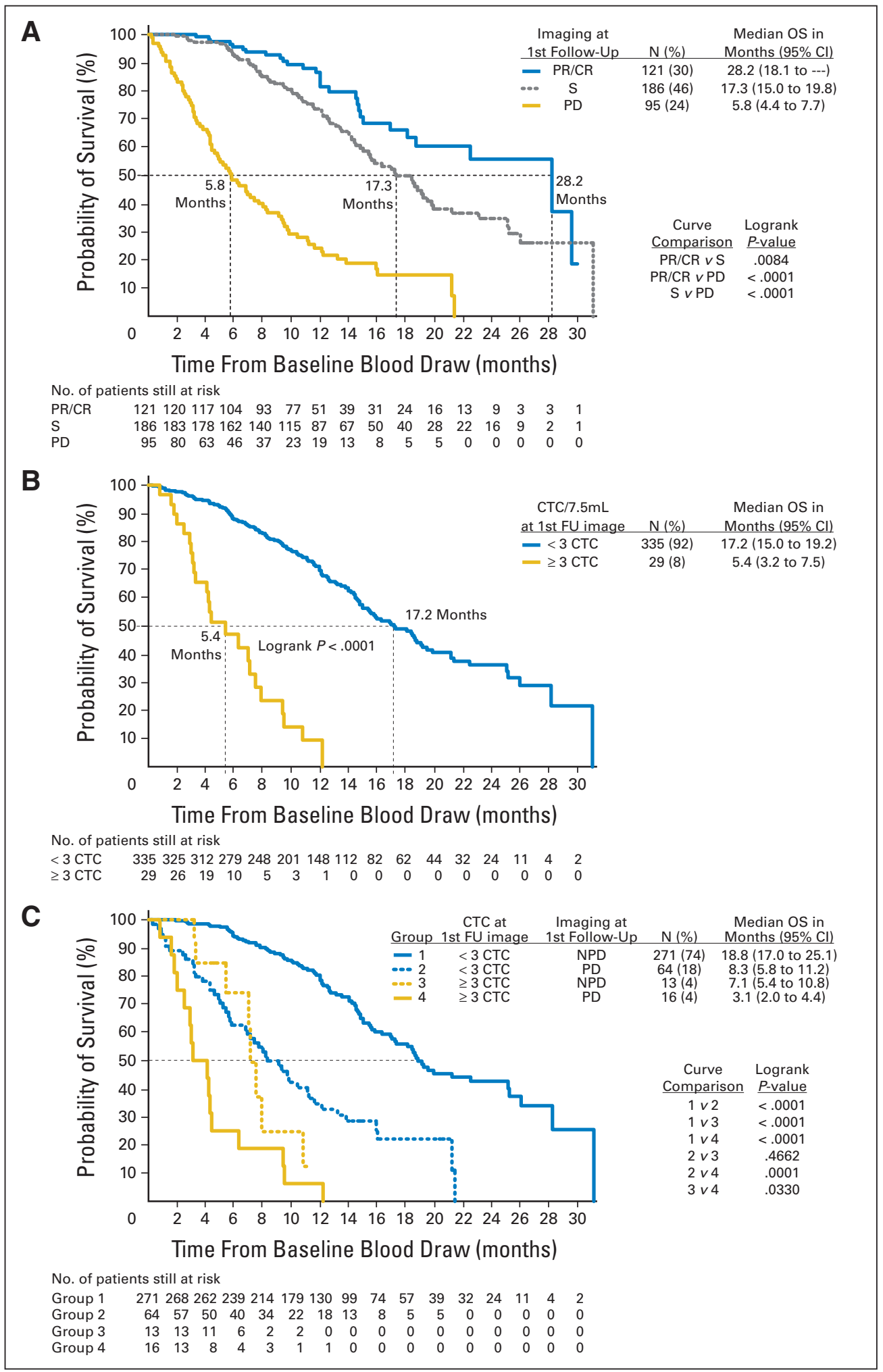

Fig 2. (A) Overall survival in metastatic colorectal cancer patients by imaging response, (B) circulating tumor cell (CTC) yield within \pm 1 month of imaging, and (C) both imaging response and circulating tumor cell yield within \pm 1 month of imaging. Overall survival (OS) values were calculated from the time of the baseline blood draws. PR, partial response; $C R$, complete response; $\mathrm{S}$, stable disease; $\mathrm{PD}$, progressive disease; FU, follow-up.

Corporation; Bruce H. Saidman, Immunicon Corporation; Kert D. Sabbath, Immunicon Corporation; Nashat Y. Gabrail, Immunicon Corporation; Joel Picus, Immunicon Corporation; Michael Morse, Immunicon Corporation; Edith Mitchell, Immunicon Corporation; Neal J. Meropol, Immunicon Corporation Expert Testimony: None Other Remuneration: None

\section{AUTHOR CONTRIBUTIONS}

Conception and design: Steven J. Cohen, Leon W.M.M. Terstappen, Neal J. Meropol 
Financial support: Leon W.M.M. Terstappen Administrative support: Leon W.M.M. Terstappen Provision of study materials or patients: Steven J. Cohen, Cornelis J.A. Punt, Nicholas Iannotti, Bruce H. Saidman, Kert D. Sabbath, Nashat Y. Gabrail, Joel Picus, Michael Morse, Edith Mitchell, Henk Tissing, Neal J. Meropol

Collection and assembly of data: Steven J. Cohen, M. Craig Miller, Henk Tissing, Leon W.M.M. Terstappen, Neal J. Meropol
Data analysis and interpretation: Steven J. Cohen, Cornelis J.A. Punt, M. Craig Miller, Gerald V. Doyle, Leon W.M.M. Terstappen, Neal J. Meropol Manuscript writing: Steven J. Cohen, Cornelis J.A. Punt, M. Craig Miller, Leon W.M.M. Terstappen, Neal J. Meropol

Final approval of manuscript: Steven J. Cohen, Cornelis J.A. Punt, Nicholas Iannotti, Bruce H. Saidman, Kert D. Sabbath, Nashat Y. Gabrail, Joel Picus, Michael Morse, Edith Mitchell, M. Craig Miller, Gerald V. Doyle, Henk Tissing, Leon W.M.M. Terstappen, Neal J. Meropol

\section{REFERENCES}

1. Jemal $A$, Siegel $R$, Ward $E$, et al: Cancer statistics, 2007. CA Cancer J Clin 57:43-66, 2007

2. Grothey A, Sargent D, Goldberg RM, et al: Survival of patients with advanced colorectal cancer improves with the availability of fluorouracilleucovorin, irinotecan, and oxaliplatin in the course of treatment. J Clin Oncol 22:1209-1214, 2004

3. Hurwitz $\mathrm{H}$, Fehrenbacher L, Novotny W, et al: Bevacizumab plus irinotecan, fluorouracil, and leucovorin for metastatic colorectal cancer. N Engl J Med 350:2335-2342, 2004

4. Cunningham $D$, Humblet $Y$, Siena $S$, et al: Cetuximab monotherapy and cetuximab plus irinotecan in irinotecan-refractory metastatic colorectal cancer. N Engl J Med 351:337-345, 2004

5. Recamier JCA: L'histoire de le Meme Maladie. Gabor vol, 1829, p 110

6. Engell HC: Cancer cells in the circulating blood: A clinical study on the occurrence of cancer cells in the peripheral blood and in venous blood draining the tumour area at operation. Acta Chir Scand 201:1-70, 1955 (suppl)
7. Allard WJ, Matera J, Miller MC, et al: Tumor cells circulate in the peripheral blood of all major carcinomas but not in healthy subjects or patients with nonmalignant diseases. Clin Cancer Res 10: 6897-6904, 2004

8. Cohen SJ, Alpaugh RK, Gross S, et al: Isolation and characterization of circulating tumor cells in patients with metastatic colorectal cancer. Clin Colorectal Cancer 6:125-132, 2006

9. Cristofanilli M, Budd GT, Ellis MJ, et al: Circulating tumor cells, disease progression, and survival in metastatic breast cancer. N Engl J Med 351:781791, 2004

10. Therasse P, Arbuck SG, Eisenhauer EA, et al: New guidelines to evaluate the response to treatment in solid tumors: European Organization for Research and Treatment of Cancer, National Cancer Institute of the United States, National Cancer Institute of Canada. J Natl Cancer Inst 92:205-216, 2000

11. Park YJ, Park KJ, Park JG, et al: Prognostic factors in 2230 Korean colorectal cancer patients: Analysis of consecutively operated cases. World J Surg 23:721-726, 1999
12. Park YJ, Youk EG, Choi HS, et al: Experience of 1446 rectal cancer patients in Korea and analysis of prognostic factors. Int J Colorectal Dis 14:101-106, 1999

13. Goldberg RM, Rothenberg ML, Van Cutsem E, et al: The continuum of care: A paradigm for the management of metastatic colorectal cancer. Oncologist 12:38-50, 2007

14. Tournigand C, Cervantes A, Figer A, et al: OPTIMOX1: A randomized study of FOLFOX4 or FOLFOX7 with oxaliplatin in a stop-and-go fashion in advanced colorectal cancer: A GERCOR study. J Clin Oncol 24:394-400, 2006

15. Maindrault-Goebel FLG, Chibaudel B, Mineur $L$, et al: Final results of OPTIMOX2, a large randomized phase II study of maintenance therapy or chemotherapy-free intervals (CFI) after FOLFOX in patients with metastatic colorectal cancer (MRC): A GERCOR study. J Clin Oncol 25:166s, 2007 (suppl; abstr 4013)

16. de Bono JS, Attard G, Adjei A, et al: Potential applications for circulating tumor cells expressing the insulin-like growth factor-l receptor. Clin Cancer Res 13:3611-3616, 2007

\section{Acknowledgment}

We thank the late Christopher Desch, MD, for his contributions in enrolling patients onto this clinical trial, and the patients who participated in this study with the understanding that they themselves would not benefit, but that the information learned could be used to help other

patients in the future.

\section{Appendix}

The Appendix is included in the full-text version of this article, available online at www.jco.org. It is not included in the PDF version (via Adobe ${ }^{\circledR} \operatorname{Reader}^{\circledR}$ ). 